\title{
A Beaconless Routing Approach for Energy Conservation in Wireless Sensor Network
}

\author{
${ }^{1}$ Patil Anil Kumar, Dr. Pm Hadalgi \\ Research Scholar Department Of Applied Electronics Gulbarga University, Gulbarga. \\ Research Guide Faculty Department Of Applied Electronics Gulbarga University, Gulbarga.
}

\begin{abstract}
Geographic Routing (GR) algorithms, require nodes to periodically transmit HELLO messages to allow neighbors know their positions (beaconing mechanism). To reduce the control overhead due to these messages, beacon-less routing algorithms have recently been proposed. However, existing beacon-less algorithms have not considered realistic physical layers. Therefore, those algorithms cannot work properly in realistic scenarios. In this paper we present a new beacon-less routing protocol EBGR. Its design is based on the new delay function to reduce collisions and duplicate messages produced during the phase of selecting the next forwarder. Through extensive simulations on NS2 we compare proposed beaconless routing protocol with $B L R$ and $C B F$ and show that our scheme is able to achieve almost perfect packet delivery ratio (like BLR) while having a low bandwidth consumption.
\end{abstract}

Keywords: Delay function, bandwidth, packet delivery ratio.

\section{Introduction}

GEOGRAPHIC routing, in which each node forwards packets only based on the locations of itself, its directed neighbors, and the destination, is particularly attractive to resource-constrained sensor networks. The localized nature of geographic routing eliminates the overhead brought by route establishment and maintenance, indicating the advantages of modest memory requirement at each node and high scalability in large distributed applications. In conventional geographic routing schemes, each node is required to maintain more or less accurate position information of all its direct neighbors, and the position of a node is made available to its direct neighbors by periodically broadcasting beacons. In WSNs with invariant or slowly changing network topology, maintaining neighbor information can greatly improve the performance because of the reusability of the maintained information and the low maintenance cost. However, in many application scenarios, WSNs are highly dynamic and the network topology may frequently change due to node mobility, node sleeping, node or link faults, etc. In highly dynamic scenarios, routing protocols based on maintaining neighbor information suffer from at least three drawbacks. First, the maintenance of neighbor information causes too much communication overhead and results in significant energy expenditure. Second, the collected neighbor information can quickly get outdated, which, in turn, leads to frequent packet drops. Third, the maintenance of neighbor information consumes memory, which is also a scarce resource in WSNs.

To overcome the drawbacks of conventional geographic routing schemes in scenarios with dynamic network topology, several beaconless geographic routing protocols [7],[5],[2],[4],[8] have been proposed. Beaconless routing schemes, in which each node forwards packets without the help of beacons and without the maintenance of neighbor information, are fully reactive. When a node has a packet to transmit, it broadcasts the packet to its neighbors. The most suitable neighbor for further relaying the packet is determined based on a contention mechanism in which each neighbor determines a proper delay for further forwarding the packet based on how well it is suited as the next-hop relay. Therefore, beaconless routing schemes are robust to topology changes since the forwarding decision is based on the actual topology at the time a packet is to be forwarded However, in most existing beaconless routing schemes such as BLR [7], CBF [5], and GDBF [4], each node forwards packets based on hop-count routing metrics (e.g., each node selects its neighbor closest to the destination as its next-hop relay). These routing metrics are simple in implementation, but they cannot guarantee energy efficiency which is a major concern in WSNs.

\section{Problem Formation}

We address the problem of providing energy-efficient beaconless geographic routing for dynamic wireless sensor networks in which network topology frequently changes over time, and present a novel routing protocol called Energy-efficient Beaconless Geographic Routing (EBGR). Without any prior knowledge of neighbors, EBGR tries to minimize the total energy consumed by delivering each packet to the sink and works as follows: each sensor node first calculates its ideal next-hop relay position based on the optimal forwarding distance in terms of minimizing the total energy consumption for delivering a packet to the sink. When a node has a packet to transmit, it first broadcasts an RTS message to detect its best next-hop relay. All suitable 
neighbors in the relay search region participate in the next-hop relay selection process using a timer-based contention mechanism. Each candidate that receives the RTS message sets a delay for broadcasting a corresponding CTS message based on a discrete delay function, which guarantees that the neighbor closest to the optimal relay position has the shortest delay. The neighbor that has the minimum delay broadcasts its CTS message first, and the other candidates snooping the CTS message notice that another node has responded the request and quit the contention process. Finally, the packet is unicasted to the established next-hop relay. If there is no node in the relay search region, the forwarding node enters into a beaconless recovery mode to recover from the local minimum.

\section{Related Work}

Beaconless routing schemes are robust to topology changes since the forwarding decision is based on the actual topology at the time a packet is to be forwarded. However, in most existing beaconless routing schemes such as BLR [7], CBF [5], and GDBF [4], each node forwards packets based on hop-count routing metrics (e.g., each node selects its neighbor closest to the destination as its next-hop relay). These routing metrics are simple in implementation, but they cannot guarantee energy efficiency which is a major concern in WSNs.

According to Contention-based forwarding [5] In this paper, propose a mechanism to perform position-based unicast forwarding without the help of beacons. In contention-based forwarding scheme (CBF) the next hop is selected through a distributed contention process based on the actual positions of all current neighbors. For the contention process, CBF makes use of biased timers. To avoid packet duplication, the first node that is selected suppresses the selection of further nodes. The general idea of CBF is to base the forwarding decision on the current neighborhood as it exists in reality and not as perceived by the forwarding node. This requires that all suitable neighbors of the forwarding node are involved in the selection of the next hop.Its main drawback is that it transmits at least two additional packets (RTF/CTF) for each forwarding of the data packet.

According to BLR[7] beacon-less routing algorithm Routing of packets in mobile ad hoc networks with a large number of nodes or with high mobility is a very difficult task and current routing protocols do not really scale well with these scenarios. The beacon-less routing algorithm (BLR) presented in this paper is a routing protocol that makes use of location information to reduce routing overhead. However, unlike other position-based routing protocols, BLR does not require nodes to periodically broadcast Hello-messages (called beaconing), and thus avoids drawbacks such as extensive interferences with regular data transmission, and performance degradation. BLR selects a Forwarding node in a distributed manner among all its neighboring nodes with having information neither about their positions nor even about their existence. Data packets are broadcasted and the protocol takes care that just one of the receiving nodes forwards the packet. Optimized forwarding is achieved by applying a concept of dynamic forwarding delay. Consequently, the node that computes the shortest forwarding delay relays the packet first. This forwarding is detected by the other nodes and suppresses them to relay the same packet any further According to GDBF[ 4] Guaranteed Delivery Beaconless Forwarding :The proposed Guaranteed Delivery Beaconless Forwarding (GDBF) protocol involves selecting the appropriate next hop by means of RTS (Ready To Send) and CTS (Clear To Beaconless Position Based Routing with Guaranteed Delivery in Sensor Networks 3 Send) packets. In greedy mode, only the forwarding neighbor sends CTS back to the node having the data packet. GDBF is a generic framework that can be applied to location based schemes. It guarantees delivery, when the underlying protocol is a guaranteed delivery protocol. The main contribution of this article is in protocol operation in recovery mode. GDBF reduces the number of messages (CTS's) sent by the neighbors of current node in recovery mode by using a special suppression scheme. The drawbacks of the GDBF are as the same as the ones in the CBF.

\section{Proposed Work}

By literature survey. We found that most of the proposed beaconless schemes employ hop-count-based routing metric, which is not efficient in terms of energy consumption. We propose a novel online geographic routing scheme called EBGR, which can provide fully stateless, energy-efficient sensor-to-sink routing at a low communication overhead without maintaining neighborhood information. We prove that EBGR is loop-free in greedy forwarding mode, We found less energy consumption for sensor-to-sink data delivery under EBGR, assuming no packet loss and no failures in greedy forwarding mode. We evaluate the performance of EBGR in scenarios: mobility, random sleeping. Simulation results show that our scheme significantly outperforms existing routing protocols in highly dynamic scenarios.

- $\quad$ each sensor node first calculates its ideal next-hop relay position based on the optimal forwarding distance in terms of minimizing the total energy consumption for delivering a packet to the sink. 
- When a node has a packet to transmit, it first broadcasts an RTS message to detect its best next-hop relay. All suitable neighbors in the relay search region participate in the next-hop relay selection process using a timer-based contention mechanism.

- Each candidate that receives the RTS message sets a delay for broadcasting a corresponding CTS message based on a discrete delay function, which guarantees that the neighbor closest to the optimal relay position has the shortest delay.

- The neighbor that has the minimum delay broadcasts its CTS message first, and the other candidates snooping the CTS message notice that another node has responded the request and quit the contention process.

- Finally, the packet is unicasted to the established next-hop relay. If there is no node in the relay search region, the forwarding node enters into a beaconless recovery mode to recover from the local minimum.

\subsection{NETWORK MODEL}

Without loss of generality, it is assumed that no two nodes locate at the same position. All sensor nodes are equipped with the same radio transceiver that enables a maximum Transmission range R. Each node knows its own location as well as the location of the sink. We use the Unit Disk Graph (UDG) communication model in the first stage of analysis.

In this model, any two nodes $u$ and $v$ can communicate with each other reliably if and only if $|u v| \leq R$, where $|\mathrm{uv}|$ is the Euclidean distance between $\mathrm{u}$ and $\mathrm{v}$. based on a realistic communication model in which data loss is estimated by packets reception rate, we extend our scheme to achieve localized energy-efficient beaconless routing in the presence of unreliable communication links.

\subsection{ENERGY MODEL}

The First Order Radio Model in [6] proposed has been widely used for measuring energy consumption in wireless communications[16],[18]. In this model, the energy for transmitting 1 bit data over distance $\mathrm{x}$ is where a11 is the energy spent by transmitter electronics, a2 is the transmitting amplifier, and) is the propagation loss exponent. The energy for receiving 1 bit data is where a12 is the energy spent by receiver electronics. Therefore, the energy consumed by relaying 1 bit data (i.e., receiving 1 bit data, and then, transmitting it over distance $\mathrm{x}$ ) is

$$
\begin{gathered}
\varepsilon_{\text {realy }}(\mathrm{x})= \\
\text { Where } \\
\text { Whe } \\
a_{1}=a_{11}+a_{12}
\end{gathered}
$$

Energy spent by transmitter $a_{11}=.07$ mill watts and energy receiver is $a_{12}=.03$ mill watts, propagation loss $\mathrm{k}<=2$

\subsection{THEORETICAL ANALYSIS}

we present extensive theoretical analysis for our algorithm based on a simplified MAC model without packet loss, the unit disk graph model without failures in greedy forwarding, and uniform node deployment. In [30], the characteristics of energy consumption for power adjusted transmission were investigated using a generalized form of the First Order Radio Model. Given a source node $u$ and a destination node v, d denotes the distance between $\mathrm{u}$ and $\mathrm{v}$, and $\varepsilon(\mathrm{d})$ represents the total energy consumed by delivering 1 bit data from $\mathrm{u}$ to $\mathrm{v}$. The following lemmas hold according to the analysis presented in [19]:

Lemma 1. If $d \leq \sqrt[k]{\frac{a_{1}}{a_{2}^{\left(1-2^{1-k}\right)}}}$

Direct transmission is the most energy-efficient way to deliver packets from $\mathrm{u}$ to $\mathrm{v}$.

Lemma 2 .if

$$
d \geq \sqrt[k]{\frac{a_{1}}{a_{2}^{\left(1-2^{1-k}\right)}}}
$$

$\varepsilon(\mathrm{d})$ is minimized when all hop distances are equal to $\frac{d}{N}$ and optimal number of hops is $\left\lfloor\frac{d}{d_{0}}\right\rfloor$ or. $\left\lceil\frac{d}{d_{0}}\right\rceil$ 


$$
d=\sqrt[k]{\frac{a_{1}}{a_{2}^{(k-1)}}}
$$

EBGR works in two modes: beaconless greedy forwarding mode and beaconless recovery mode. In the former mode, only the nodes in the relay search region (see Fig. 1) of the forwarder are candidates for further forwarding the packet, and the forwarder chooses the neighbor closest to its optimal relay position as its nexthop relay using the RTS/CTS handshaking mechanism. In this way, each packet is expected to be delivered along the minimum energy route from the source to the sink. If there is no node in the relay search region, the forwarder enters into beaconless recovery mode.

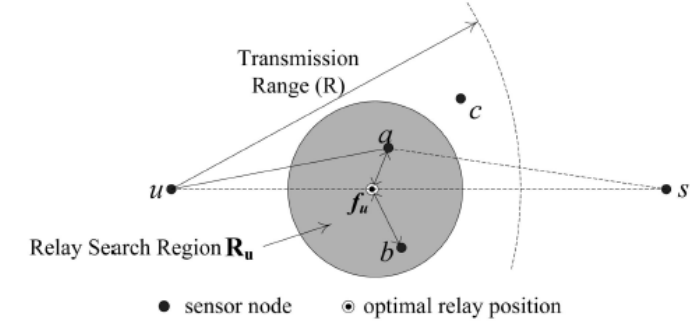

Fig. 1. Greedy forwarding in EBGR.

\section{Relay Search Region}

Since the best next-hop relay for any node $\mathrm{u}$ is the neighbor closest to its ideal relay position fu, there is no need for all neighbors of node $u$ to participate in the contention for acting as the next-hop relay. In EBGR, each node has a relay search region which is defined as follows: Definition 2. Given any node u, its next-hop relay search region, denoted by, is $R u$ defined as the disk centered at $u$ 's ideal next-hop relay position $f u$ with radius $R_{S}(u)$ where

$$
R_{S}(\mathrm{u}) \leq\left|u f_{u}\right|=d_{0}
$$

For any node $\mathrm{u}$, only the neighbors in its relay search region $R u$ are candidates for further forwarding the packets transmitted from node $u$. The concept of relay search region is introduced to prohibit the unsuitable neighbors from participating the relay contention procedure.

\subsection{Beaconless Greedy Forwarding}

The nodes in the relay search region (see Fig. 1) of the forwarder are candidates for further forwarding the packet, and the forwarder chooses the neighbor closest to its optimal relay position as its next-hop relay using the RTS/CTS handshaking mechanism. In this way, each packet is expected to be delivered along the minimum energy route from the source to the sink. Since the best next-hop relay for any node $u$ is the neighbor closest to its ideal relay position $f u$, there is no need for all neighbors of node $u$ to participate in the contention for acting as the next-hop relay. For any node $u$, only the neighbors in its relay search region $R u$ are candidates for further forwarding the packets transmitted from node $u$. The concept of relay search region is introduced to prohibit the unsuitable neighbors to participate in the relay contention procedure. Given any node $u$, let $|u s|$ be the distance from node $u$ to the sink $s$. Node $u$ first calculates $|u s|$ since it has the knowledge of its own position as well as the position of the sink. If the sink is in u's transmission

$$
|u s| \leq \sqrt[k]{\frac{a_{1}}{a_{2}^{\left(1-2^{1-k}\right)}}}
$$

Node $u$ transmits its packets directly to the sink because relaying the packets by some intermediate nodes is no more energy-efficient than direct transmission. Otherwise, node $u$ detects it best next-hop relay based on the procedure given as follows: $\operatorname{Let}\left(x_{u}, y_{u}\right)$ and $\left(x_{s}, y_{s}\right)$ be the coordinates of node $\mathrm{u}$ and the sink $s$, respectively. The location of $f_{u}$, denoted by $\left(x_{u_{0}}, y_{u_{0}}\right)$, can be computed as follows:

$x_{u_{0}} \equiv x_{u}-\frac{d_{0}}{|u s|}\left(x_{u}-x_{s}\right)$

$y_{u_{0}} \equiv y_{u}-\frac{d_{0}}{|u s|}\left(y_{u}-y_{s}\right)$

When node $u$ has a packet to transmit, it broadcasts an RTS message, which also contains the location of its ideal next-hop relay position as well as the radius of its relay search region, to detect its best next-hop relay. For any neighbor $w$ that receives the RTS message from node $u$, it first checks if it falls in $R u$. If $\mathrm{w} \neq R u$, the RTS message is simply discarded. Otherwise, node $w$ generates a CTS message which also contains its own location and sets a proper delay, denoted by $\delta w \rightarrow u$, for broadcasting the CTS message based on a discrete delay function 
given in next section. If node w overhears a CTS message broadcasted by another candidate before $\delta w \rightarrow u$ is due, node $w$ cancels broadcasting its own CTS message; otherwise, node $w$ broadcasts its CTS message when $\delta w \rightarrow u$ is due. When node $u$ receives the CTS message from its neighbor $w$, the next-hop relay for $u$, denoted by relay $(u)$, is updated if relay $(u)$ is null or $|w f u|<\mid$ relay $(u) f u \mid$. Finally, node $w$ unicasts its packet to its next-hop relay relay $(u)$. Nodes located within this forwarding area apply Discrete Delay [8] prior to relaying the packet, whereas nodes outside this area drop the received packet. The value of the Discrete Delay [0, Max_Delay] depends on the relative position coordinates of current, previous, and destination node. Eventually, the node which computed the shortest Discrete Delay forwards the packet first. Every node in the forwarding area detects the further relaying of the packet and cancels its scheduled transmission of the same packet. Furthermore, passive acknowledgments are used.

The previous transmitting node also detects the further relaying of the packet and thus concludes that it was successfully received by another node. Thereby, acknowledgments on the MAC-layer can be avoided. (For example in IEEE 802.11, there are even no acknowledgments provided for broadcast packets.) The algorithm continues until the destination is reached. The only node that has to send an acknowledgement is the destination node since it does not relay the packet any further.

\subsection{BEACONLESS RECOVERY}

When node $u$ broadcasts an RTS message to detect its best next-hop relay, it sets its timer to tmax and starts the timer. tmax is large enough to guarantee that node $u$ can receive the CTS message from the furthest neighbor in $\mathrm{Ru}$ before the timer is expired. If node $\mathrm{u}$ receives no CTS message till the timer is expired, it assumes that there is no neighbor in its relay search region. To recover from the local minimum, the beaconless angular relaying proposed in [17] is employed in EBGR. The angular relaying algorithm works in two phases: selection phase and protest phase. In the selection phase, the forwarder u broadcasts an RTS message to its neighbors, and the neighbors answer with CTS messages in counterclockwise order according to an angularbased delay function. After the first candidate w answers with a valid CTS, the protest phase begins. First, only the nodes in $N_{G G}(v, w)$ (i.e., the Gabriel circle having uw as diameter) are allowed to protest. If a node $\mathrm{x}$ protests, it automatically becomes the next-hop relay. After that, only nodes in $N_{G G}(v, w)$ are allowed to protest. Finally, the forwarder sends the packet to the selected (first valid or last protesting) candidate.

\section{Results}

Fig2 Packet Delivery Ratio: It is defined by a factor of number of packets received by number of packets transmitted. v/s node density we compare the results of direct, intermediate relay, EBGR modified, EBGR performance and beaconless recovery , fig3 energy consumption by data transmission v/s nodes density comparing the energy consumption in EBGR modified, EBGR performance.fig 4 latency v/s nodes density

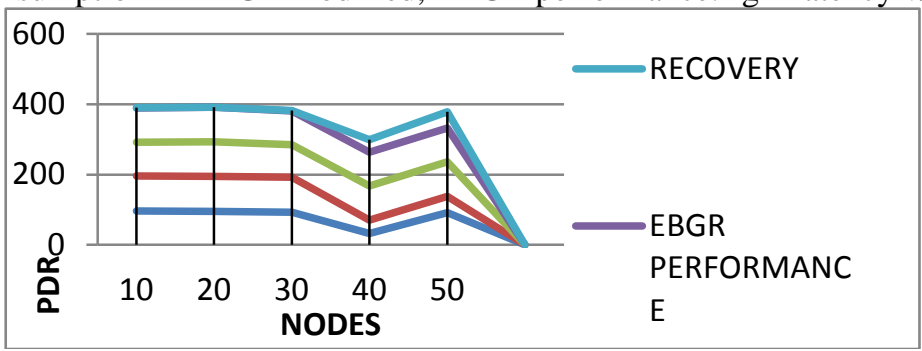

Fig2.packet deliver ratio(joules) v/s node densityIt can be seen that the Packet delivery ratio of EBGR with recovery is better than other peers of geographic routing. Fundamentally the algorithm poffers quick recovery from route and path loss. Therefore the system is better.

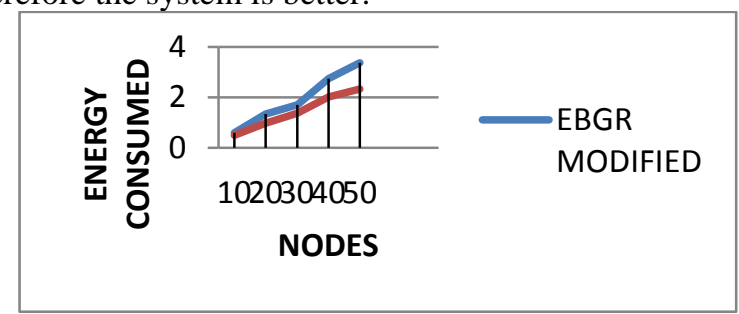

Fig3: energy consumed (joules) vls nodes

Average energy consumed of EGBR is better than EGBR modified. This is partially due to lesser packet success rate of EGBR. As the modified version offers better resilence against losses, the system transmits more packets and hence losses are more. 


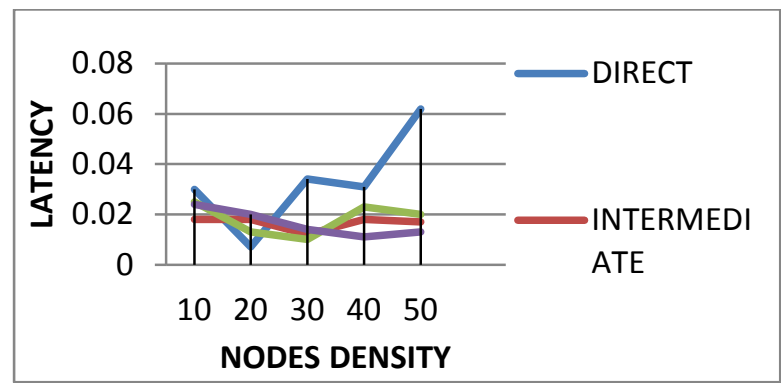

Fig4: latency v/s node density

It can be seen that for average number of nodes ( nodes bellow 30) EGBR modified's latency is better. This is because for the same range, pdr of all the systems are higher. Hence it can be stated that for transmitting packets with equal success, performance of the modified version is better. But as the nodes increases, EGBR modified gives better PDR hence more packets are transmitted which results in higher delay.

\section{Conclusion}

Providing energy-efficient routing is an important issue in the design of WSNs. In this paper, we propose a novel energy-efficient beaconless geographic routing protocol EBGR which takes advantages of both geographic routing and power-aware routing to provide loop-free, stateless, and energy-efficient sensor-to-sink routing in dynamic WSNs. The performance of EBGR is evaluated through simulations. Simulation results show that our protocol consumes significantly less energy than routing protocols based on neighborhood maintenance in highly dynamic scenarios. There are some interesting future research directions regarding the concept of energy-efficient geographical routing in WSNs. By taking the residual energy into account for making forwarding decision, our scheme can be extended to alleviate the unbalanced energy consumption in the network while still guarantee that the total energy consumption for sensor-to-sink data delivery is bounded. Another extension is to integrate other energy conserving schemes such as data aggregation to further Reduce energy consumption and maximize network lifetime. It is also interesting to extend our scheme to networks with heterogeneous propagation properties There are some interesting future research directions regarding the concept of energy-efficient geographical routing in WSNs. By taking the residual energy into account for making forwarding decision, our scheme can be extended to alleviate the unbalanced energy consumption in the network while still guarantee that the total energy consumption for sensor-to-sink data delivery is bounded. Another extension is to integrate other energy conserving schemes such as data aggregation to further Reduce energy consumption and maximize network lifetime. It is also interesting to extend our scheme to networks with heterogeneous propagation properties

[1] http://www.omnetpp.org/index.php, 2009.

\section{References}

[2] B. Blum, T. He, S. Son, and J. Stankovic, "IGF: A State-Free Robust Communication Protocol for Wireless Sensor Networks," Technical Report CS-2003-11, Univ. of Virginia, 2003.

[3] J.-H. Chang and L. Tassiulas, "Maximum Lifetime Routing in Wireless Sensor Networks," IEEE/ACM Trans. Networking, vol. 12, no. 4, pp. 609-619, Aug. 2004.

[4] M. Chawla, N. Goel, K. Kalaichelvan, A. Nayak, and I. Stojmenovic, "Beaconless Position Based Routing with GuaranteedDelivery for Wireless Ad-Hoc and Sensor Networks," Proc.FIP Int'l Federation for Information Processing World ComputerCongress, pp. 61-70, 2006.

[5] H. Fu“ßler, J. Widmer, M. Ka“semann, M. Mauve, and H.Hartenstein, "Contention-Based Forwarding for Mobile Ad Hoc Networks," Ad Hoc Networks, vol. 1, pp. 351-369, 2003.

[6] W.R. Heinzelman, A. Chandrakasan, and H. Balakrishnan, "Energy-Efficient Communication Protocol for Wireless Microsensor Networks," Proc. 33rd Hawaii Int'l Conf. System Sciences, pp. 4-7, 2000.

[7] M. Heissenbu" ttel, T. Braun, T. Bernoulli, and M. Wa"lchli, "BLR: Beacon-Less Routing Algorithm for Mobile Ad Hoc Networks," Computer Comm., vol. 11, pp. 1076-1086, 2004.

[8] H. Kalosha, A. Nayak, S. Ru" hrup, and I. Stojmenovic, "Select-and- Protest-Based Beaconless Georouting with Guaranteed Delivery in Wireless Sensor Networks,” Proc. IEEE INFOCOM, pp. 346-350, 2008.

[9] K. Kalpakis, K. Dasgupta, and P. Namjoshi, "Efficient Algorithms for Maximum Lifetime Data Gathering and Aggregation in Wireless Sensor Networks," Computer Networks, vol. 42, pp. 697-716, 2003.

[10] J. Kuruvila, A. Nayak, and I. Stojmenovic, "Hop Count Optimal Position Based Packet Routing Algorithms for Ad Hoc Wireless Networks with a Realistic Physical Layer,” Proc. First IEEE Int'1Conf. Mobile Ad-Hoc and Sensor Systems (MASS), pp. 398-405, 2004.

[11] S. Lee, B. Bhattacharjee, and S. Banerjee, "Effcient Geographic Routing in Multihop Wireless Networks," Proc. ACM MobiHoc, pp. 230-241, 2005.

[12] T. Melodia, D. Pompili, and I.F. Akyildiz, "Optimal Local Topology Knowledge for Energy Efficient Geographical Routing in Sensor Networks," Proc. IEEE INFOCOM, 2004.

[13] K. Seada, M. Zuniga, A. Helmy, and B. Krishnamachari, "Energy-Efficient Forwarding Strategies for Geographic Routing in Lossy Wireless Sensor Networks,” Proc. Second Int'l Conf. Embedded Networked Sensor Systems (SenSys), pp. 108-121, 2004. 
[14] S. Singh, M. Woo, and C.S. Mghavendra, "Power-Aware Routing in Mobile Ad Hoc Networks," Proc. ACM MobiCom, pp. 181-190, 1998.

[15] I. Stojmenovic, "Localized Network Layer Protocols in Wireless Sensor Networks Based on Optimizing Cost over Progress Ratio,"IEEE Network, vol. 20, no. 1, pp. 21-27, Jan./Feb. 2006.

[16] I. Stojmenovic and X. Lin, "Power-Aware Localized Routing in Wireless Networks," IEEE Trans. Parallel and Distributed Systems, vol. 12, no. 11, pp. 1122-1133, Nov. 2001.

[17] H. Takagi and L. Kleinrock, "Optimal Transmission Ranges for Randomly Distributed Packet Radio Terminals," IEEE Trans.Comm., vol. COM-32, no. 3, pp. 246- 257, Mar. 1984

[18] S. Wu and K.S. Candan, "GPER: Geographic Power Efficient Routing in Sensor Networks

[19] I. Stojmenovic and X. Lin, "Power-Aware Localized Routing in Wireless Networks," Proc. 14th Int'1 Parallel and Distributed 